\section{Qualitätssandards für die mpMRT der Prostata}

Wie bereite ich eine multiparametrische MRT (mpMRT) der Prostata vor und wie sollte das Untersuchungsprotokoll am besten aufgebaut sein? Die Empfehlungen dazu hat die Arbeitsgemeinschaft Uroradiologie und Urogenitaldiagnostik in der Deutschen Röntgengesellschaft (DRG) in Abstimmung mit dem DRG-Vorstand und dem Vorstand des Berufsverbands der Deutschen Radiologen (BDR) im März dieses Jahres überarbeitet und aktualisiert. Wir haben bei dem AG-Vorsitzenden PD Dr. Lars Schimmöller, der als Autor an der Aktualisierung mitgewirkt hat, genauer nachfragt.

Herr Doktor Schimmöller, die AG Uroradiologie und Urogenitaldiagnostik der Deutschen Röntgengesellschaft hat die Empfehlungen aus dem Jahr 2017 zur Vorbereitung und Durchführung der MRT überarbeitet und aktualisiert. Weshalb war dies notwendig?

Die multiparametrische MRT (mpMRT) der Prostata, oder auch „MR-Prostatographie“, hat sich erfreulicherweise in den vergangenen Jahren national und international fest etabliert. Durch den technischen und wissenschaftlichen Fortschritt war es erforderlich, die Empfehlungen der Deutschen Röntgengesellschaft (DRG) aus dem Jahr 2017 kritisch zu überprüfen und unter der Berücksichtigung der 2019 aktualisierten internationalen PI-RADS-Empfehlungen (Version 2.1) anzupassen. Zudem gab es die Zielsetzung, die Empfehlungen gleichzeitig auch mit den bis dato teils abweichenden Parameterangaben des Berufsverbandes der Deutschen Radiologen (BDR) zu harmonisieren und als Referenz für das Update der S3-Leitlinie zum Prostatakarzinom fertigzustellen. Von Februar 2020 bis Januar 2021 haben wir in über 20 Telefonkonferenzen die Empfehlungen komplett überarbeitet und unter den elf Ko-Autoren, welche Mitglied der DRG und teils auch des BDR sind, neu diskutiert. Geleitet und organisiert wurde die Überarbeitung von Professor Tobias Franiel (Universitätsklinikum Jena), der letztendlich die auch teils unterschiedlicheren Expertenmeinungen und abschließend die Publikation koordiniert hat. Das nun vorliegende, umfassende Ma- nuskript dient somit als „deutsche“ Referenz zur Vorbereitung und Durchführung der mpMRT der Prostata und ist gleichzeitig auch Referenz der in der aktualisierten S3Leitlinie (Mai 2021; Version 6.0) genannten geltenden mpMRT-Qualitätsstandards.

\section{Welche Änderungen beziehungsweise Neuerungen sind aus Ihrer Sicht beson- ders wichtig?}

Wir haben im Rahmen der Publikation analog zur Vorpublikation alle wichtigen Fakten in einer Tabelle für die Anwender:innen zusammengefasst und kondensiert. Grob gliedern sich die Empfehlungen in relevante anamnestische Angaben (Teil 1), Untersuchungsterminierung und -vorbereitung (Teil 2), einen ausführlichen technischen Teil zum Untersuchungsprotokoll (Teil 3) und abschließend die Empfehlungen zur MRT-(inbore)-Biopsie (Teil 4). So halten wir es für sehr wichtig und erforderlich, dass ein aktueller PSA-Wert zu jeder ProstataMRT-Untersuchung vorhanden ist, bestenfalls sogar ein PSA-Wert-Verlauf. Auch Informationen zu vorherigen Biopsien und Therapien, aber auch Implantaten sollten angefragt werden beziehungsweise vorliegen. Hieraus lässt sich nämlich eine klinische Einordnung ableiten und auch die PSA-Dichte mit dem in der MRT bestimmten Prostatavolumen berechnen, was für die Konklusivität der MRT-Bilder schlussendlich genutzt werden sollte. Neu ist unter anderem die dezidierte Auseinandersetzung mit der Angulation der axialen beziehungsweise paraaxialen Bilder. Aber nicht nur zertifizierten Radiolog:innen empfehle ich, die Publikation in Gänze zu lesen und als Referenz zu nutzen. Alles in allem ist das Ziel aber natürlich, dass aus den Empfehlungen am Ende in der Breite gute Bilder zur Prostatakarzinomdiagnostik erstellt werden. Die Vorgaben sind hierbei als detaillierte Eckpfeiler zu sehen, eine gerätespezifische Optimierung sollte und muss unter Berücksichtigung der Empfehlung dennoch stets erfolgen. Eine reine Übertragung der Parameter führt natürlich nicht automatisch zum besten Resultat, hier ist meist noch ein „Feintuning“ nötig. Nur durch eine sehr gute diagnostische Qualität können wir auch höchste Sicherheit in der Prostatakarzinomdiagnostik geben, hier

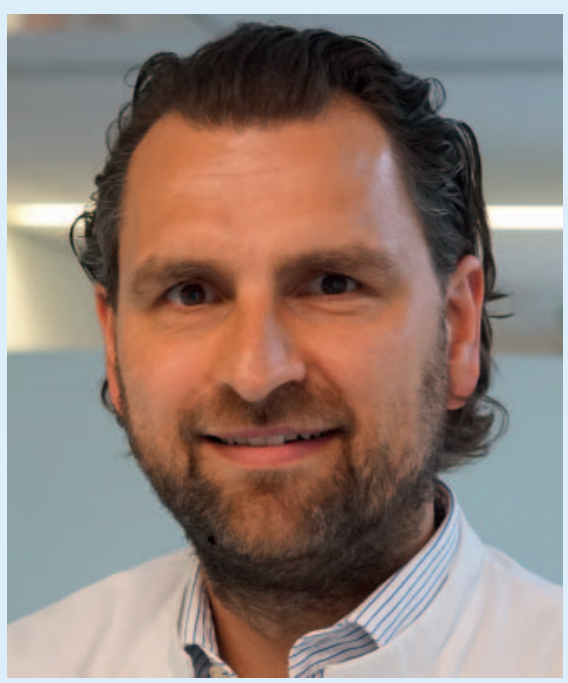

PD Dr. Lars Schimmöller

wäre ein Stichwort zum Beispiel „PI-QUAL“ (Prostate Imaging Quality; Giganti F et al. 2020 Eur Urol Oncol). Bei nicht optimaler Qualität sinkt der negativ prädiktive Wert (sicherer Ausschluss von klinisch relevanten Karzinomen) und letztendlich auch der positiv prädiktive Wert (sichere Detektion von klinisch relevanten Karzinomen), was stets bei der Befundung berücksichtig werden muss.

Haben die aktualisierten Empfehlungen Auswirkungen auf die Inhalte der Spezialzertifizierung mpMRT Prostata der AG Uroradiologie und Urogenitaldiagnostik und wenn ja, welche genau?

Die aktualisierten Empfehlungen ersetzen natürlich die der alten Publikation von 2017. Genau wie die Prostata-MRT sich weiterentwickelt, erfährt auch die Zertifizierung eine regelmäßige Re-Evaluation und Aktualisierung. Die aktuellen Antragsformulare wurden zuletzt Anfang dieses Jahres - mit Stand vom 12.01.2021 - unter anderem an die Vorgaben aus der neuen Empfehlung angepasst und fordern beziehungsweise überprüfen entsprechende Protokollparameter. Übergeordnetes Ziel ist es, den Kolleg:innen mit den aktualisierten Empfehlungen eine Handlungsempfehlung an die Hand zu geben und über die Zertifizierung einen Anreiz zur spezialisierten Weiterbildung sowie eine entsprechende Wiederspiegelung ihrer erworbenen Expertise zu schaffen. So können 
wir als radiologische Fachgesellschaft auch in der Breite zuverlässig eine geforderte, entsprechende Qualität liefern und abbilden.

Die aktualisierten Empfehlungen der DRG und des BDR zur Vorbereitung und Durchführung der mpMRT der Prostata können Sie hier nach- lesen: https://www.thieme-connect.de/pro ducts/ejournals/pdf/10.1055/a-1406-8477. pdf?articleLanguage $=$ de

Franiel T, Asbach P, Beyersdorff D, Blondin $D$, Kaufmann S, Mueller-Lisse UG, Quentin M, Rödel S, Röthke M, Schlemmer HP, Schimmöl- ler L. mpMRI of the Prostate (MR-Prostatography): Updated Recommendations of the DRG and BDR on Patient Preparation and Examination Protocol. Fortschr Röntgenstr 2021; 193: 763-776 